\title{
Factors Affecting the Quality of Life in Esophageal Cancer Patients
}

\author{
Dan Dan Wang ${ }^{1}$, Yu Jia Wei ${ }^{1}$, Hong Zhen $\mathrm{Du}^{1}$, Ying Xie ${ }^{1}$, Bin $\mathrm{Luo}^{1}$, Huan Yu Hu${ }^{1}$, Hong Xia Xu', \\ Chun Hua Song ${ }^{3}$, Han Ping Shi ${ }^{4}$, Zeng Ning $\mathrm{Li}^{1}$
}

The Investigation on Nutrition Status and Clinical Outcome of Common Cancers (INSCOC) Group; ${ }^{I}$ Department of Nutrition, the First Hospital of Hebei Medical University, Shijiazhuang, Hebei 050031, China; ${ }^{2}$ Department of Clinical Nutrition, Daping Hospital, Third Military Medical University, Chongqing 400042, China; ${ }^{3}$ Department of Epidemiology and Health Statistics, School of Public Health, Zhengzhou University, Zhengzhou, Henan 450001, China; ${ }^{4}$ Department of Clinical Nutrition, Beijing Shijitan Hospital, Capital Medical University, Beijing 100038, China

\begin{abstract}
Objective The aim of the study was to assess the quality of life (QoL) of patients with esophageal cancer, and to explore the main socioeconomic factors that affect the QoL. Method A multicenter cross-sectional study design was used. Data were collected using scales including the interviewer-administered general situation questionnaire designed by the authors, the Quality of Life Questionnaire-core 30 Version 3 in Chinese (QLQ-C30), and the Patientgenerated Subjective Global Assessment (PG-SGA) from a nationwide survey conducted previously in China. The role of each covariate in the full model was assessed using the QoL as the primary outcome in a multilevel ordinal logistic regression analysis. Results In total, 1223 patients were included, among whom 1009 (82.5\%) were males, and 504 $(41.2 \%)$ were older than 65 years old. The findings indicate that patients with esophageal cancer in China face a poor quality of life with a mean global quality of life scored of 58.33 (66.67). The most significant factors influencing quality of life were the nutritional support, PG-SGA scores and level of education. Compared with patients who had a PG-SGA score $\geq 9$, patients with scores between 0-1 had significantly better scores in five scales: the PF (physical functioning), RF (role functioning), CF (cognitive functioning), EF (emotional functioning) and SF (social functioning) (OR: 4.75, 4.84, 2.12, 2.81, and 3.00). Conclusion Nutritional support and PG-SGA scores significantly influenced the quality of life of patients with esophageal cancer. It appears that most patients with esophageal cancer are in urgent need of symptom alleviation and/or concurrent intervention.
\end{abstract}

Key words: Esophageal cancer; Quality of life; Malnutrition; Nutritional support; PG-SGA

\section{Introduction}

Esophageal cancer is the eighth most common cancer worldwide $(456,000$ new cases in $2012 ; 3 \%$ of all cancers in 2012) [1] and is known for its marked variation by geographic region, ethnicity, and gender [2]. The incidence rates are significantly higher among men than women. It is rare among persons aged $<50$ years [2], and incidence rates gradually increase with age. It is expected that the incidence of esophageal cancer will continue to rise [3] with the continuous development of industrialization and urbanization in China [4], becoming a major threat to human health. Because esophageal cancer is usually diagnosed in advanced stages [5], it has ranked sixth among cancer deaths worldwide (400,000 deaths in 2012) [6]. In the past decade, the patient quality of life (QoL) has been an important focus for evaluating the outcomes of therapy for esophageal cancer, particularly due to its low cure rate despite aggressive treatment [7].

The QoL is a concept introduced by the European

Corresponding author: Zeng Ning Li, Department of Nutrition, The First Hospital of Hebei Medical University, 89 Donggang Road, Shijiazhuang, Hebei 050031, China; Tel: +86 1863388 9888; Fax: +86 3118591 7290; Email: lizengning@126.com
Organization for Cancer Research, which is based on the subjective feelings of the individuals involved and can be measured by a validated questionnaire known as the QoL Questionnaire Core 30 (QLQ-C30) [8]. QLQ-C30 includes 5 functional scales, 3 symptom scales, 6 single-item questions and questions about the global QoL. Quality of Life questionnaires can be used as screening tools to identify those who might benefit from psycho-social intervention [9]. The QoL of patients is significantly affected by esophageal cancer. There is also evidence indicating that a poor QoL is associated with poorer long-term survival in esophageal cancer patients [10]. Thus, it is important to identify the most prominent factors influencing QoL to provide better care that can improve the QoL among patients with esophageal cancer.

\section{Material and Methods Study design}

This cross-sectional study was conducted to assess the QoL of patients with esophageal cancer, and to explore the main factors that affect the QoL of these patients. After receiving approval from the Research Ethics Board of the First Hospital of Hebei Medical University, face-to-face interviews are carried out from October 2011 to June 2016 
for a total of 1508 patients with malignant tumors from 52 hospitals in China.

\section{Subjects}

Patients pathologically diagnosed with esophageal cancer and aged at least 18 years old were eligible for inclusion in the study. Data collection was attributed to individual patients rather than individual admissions. The exclusion criteria were: 1) patients who were unwilling to participate in the study, 2) patients aged $<18$ or $>90$ years, 3 ) patients with physical or cognitive impairment, 4) patients with AIDS and recipients of organ transplants. Only the patients who met the inclusion criteria and provided signed consent were invited to participate in this study.

\section{Instruments}

A set of questionnaires was used to obtain information from the subjects. Demographic data were obtained through a questionnaire (including the patient's name, sex, age, education level, cancer stage, pathological type, etc.) designed by the authors. The PG-SGA [11], which was developed especially for cancer patients, covers seven items: body weight, food intake, symptoms, activities and function, disease and its relation to nutritional requirements, metabolic demand and the results of a physical examination to produce a global assessment of well-nourished or anabolic (A), moderate or suspected malnutrition (B) or severe malnutrition (C). For the global and functional scales, a higher score indicates better global and physical functioning; while for the symptom scale, a higher score indicates worse symptoms. The European Organization of Research and Treatment of Cancer Quality of Life questionnaire (EORTC QLQ-C30) was used to assess the patient quality of life. This instrument is a 30-item multi-dimensional cancer-specific questionnaire developed to assess the QoL of cancer patients in the following domains: functional scales, symptom scales, global QoL and single items. It contains five functional scales (physical, role, cognitive, emotional and social), symptom scales (appetite loss, nausea, vomiting, pain, dyspnea, constipation, diarrhea, fatigue, insomnia, financial difficulties) with one global health scale (GHS) and six single items assessing symptoms and the financial impact of the disease.

\section{Statistical analysis}

Information collected from patients at the interview included their age, sex, occupation, permanent residence, educational background, type of health insurance, staging of cancer, pathological type and PG-SGA. A numeric score was calculated in each dimension area of the QLQ-C30. Descriptive data were presented using medians (2nd quartile). For the multivariate analysis, the role of each covariate in the full model was assessed using the QoL as the primary outcome in a multilevel ordinal logistic regression analysis. The SPSS software (SPSS Inc., version 13.0) was utilized for the analyses. Values of $P<0.05$ were considered to be statistically significant.

\section{Results \\ Patient characteristics}

Interviews were conducted as face-to-face inquiries by professionals who were trained uniformly between June 2013 and June 2014. A total of 1508 patients were enrolled in the study, and 1223 had complete baseline information. Among these 1223 adults age $>18$ years who resided in China, there were 1009 (82.5\%) men and $214(17.5 \%)$ women. Interviews were conducted in Chinese. Data were weighted to reflect their proportions in the population. Weighted variables included gender, age, permanent residence, health insurance, occupation, education, staging of cancer, and several other variables, the details of which are shown in Tables 1 and 2.

Table 1 Basic information of the study patients with esophageal cancer $(n=1223)$.

\begin{tabular}{|c|c|c|}
\hline Characteristic & $n$ & $\%$ \\
\hline \multicolumn{3}{|l|}{ Gender } \\
\hline Male & 1009 & 82.5 \\
\hline Female & 214 & 17.5 \\
\hline \multicolumn{3}{|l|}{ Age (years) } \\
\hline$<65$ & 719 & 58.8 \\
\hline$\geq 65$ & 504 & 41.2 \\
\hline \multicolumn{3}{|l|}{ Permanent residence } \\
\hline Urban $^{1}$ & 635 & 51.9 \\
\hline Rural $^{2}$ & 588 & 48.1 \\
\hline \multicolumn{3}{|l|}{ Health insurance } \\
\hline Medicaid & 1087 & 88.9 \\
\hline Self-pay & 136 & 11.1 \\
\hline \multicolumn{3}{|l|}{ Occupation } \\
\hline Worker $^{3}$ & 74 & 6.1 \\
\hline Farmer & 523 & 42.8 \\
\hline Cadre $^{4}$ & 181 & 14.8 \\
\hline Retiree + others $^{5}$ & 445 & 36.4 \\
\hline \multicolumn{3}{|l|}{ Education } \\
\hline Primary school or below & 574 & 47.0 \\
\hline Junior/ high school or above & 569 & 46.5 \\
\hline University & 80 & 6.5 \\
\hline
\end{tabular}

${ }^{1}$ The patient's residence is municipalities or established towns; ${ }^{2}$ Patients are non-urban residence; ${ }^{3}$ Personal files are managed by the ministry of human resources and social security; ${ }^{4}$ Personal files are managed by the city's human resources department; ${ }^{5}$ Retiree or a patient who has worked in any job

\section{Health-related quality of life in patients with esophageal cancer}

Esophageal cancer seriously affects the QoL of patients. The scores of the physical functioning (PF), role functioning 
(RF), cognitive functioning (CF), emotional functioning (EF) and social functioning (SF) scales were 86.67 (100.00), 83.33 (100.00), 91.67 (100.00), 100.00 (100.00) and 66.67 (100.00), respectively. The scores of three symptom domains (fatigue, nausea and vomiting, pain) were 22.22 (33.33), 0.00 (16.67) and 0.00 (33.33), respectively. Table 3 displays the values for each domain of the QLQ-C30 questionnaire. A multivariate ordinal logistic regression analysis of the main factors that affect the QOL showed that the general quality of life among patients with junior /high school or above-level education was higher than that of the patients with a primary school education or lower (OR: 1.44). Patients with nutritional support also had better general quality of life scores (OR: 1.91), and the PG-SGA scores were correlated with the quality of life, with odds ratios of 4.85, 2.79, 2.30, and 1.0, respectively, for PG-SGA scores of $0-1,2-3$, and 4-8 and $>9$ (Table 4). Variables are represented by $\mathrm{M}(\mathrm{Q})$.

Table 2 The clinical characteristics in patients with esophageal cancer $(n=1223)$.

\begin{tabular}{|c|c|c|}
\hline Characteristic & $n$ & $\%$ \\
\hline \multicolumn{3}{|l|}{ Staging } \\
\hline 0 & 459 & 37.5 \\
\hline I & 70 & 5.7 \\
\hline II & 204 & 16.7 \\
\hline III & 300 & 24.5 \\
\hline IV & 190 & 15.5 \\
\hline \multicolumn{3}{|l|}{ Pathological typing } \\
\hline Squamous cell & 1033 & 84.5 \\
\hline Adenocarcinoma & 79 & 6.5 \\
\hline Small cell & 21 & 1.2 \\
\hline Mucinous & 2 & 0.2 \\
\hline Other & 88 & 7.2 \\
\hline \multicolumn{3}{|l|}{ Cancer treatment } \\
\hline Surgery & 270 & 22.1 \\
\hline Chemo-radiotherapy & 332 & 27.1 \\
\hline Chemotherapy + surgery & 291 & 23.8 \\
\hline No treatment & 180 & 14.7 \\
\hline Other & 150 & 12.3 \\
\hline \multicolumn{3}{|l|}{ PG-SGA } \\
\hline $0 \sim 1$ & 73 & 6.0 \\
\hline $2 \sim 3$ & 96 & 7.9 \\
\hline $4 \sim 8$ & 179 & 14.6 \\
\hline$\geq 9$ & 875 & 71.5 \\
\hline \multicolumn{3}{|l|}{ NRS 2002} \\
\hline$<3$ & 612 & 50.0 \\
\hline$\geq 3$ & 608 & 49.7 \\
\hline Missing & 3 & 0.03 \\
\hline
\end{tabular}

Table 3 The quality of life in esophageal cancer patients $(n=1223)$.

\begin{tabular}{|c|c|c|c|c|}
\hline Variable & $\begin{array}{c}\text { 1st } \\
\text { quartile }\end{array}$ & $\begin{array}{c}\text { 2nd } \\
\text { quartile }^{1}\end{array}$ & $\begin{array}{c}\text { 3rd } \\
\text { quartile }\end{array}$ & $\begin{array}{c}\text { 4th } \\
\text { quartile }\end{array}$ \\
\hline \multicolumn{5}{|l|}{ General quality of life } \\
\hline Global quality of life & 50.00 & 58.33 & 66.67 & 100.00 \\
\hline \multicolumn{5}{|l|}{ General function } \\
\hline Physical & 73.33 & 86.67 & 100.00 & 100.00 \\
\hline Role & 66.67 & 83.33 & 100.00 & 100.00 \\
\hline Emotional & 75.00 & 91.67 & 100.00 & 100.00 \\
\hline Cognitive & 83.33 & 100.00 & 100.00 & 100.00 \\
\hline Social & 66.67 & 66.67 & 100.00 & 100.00 \\
\hline \multicolumn{5}{|l|}{ General symptom } \\
\hline Fatigue & 0.00 & 22.22 & 33.33 & 100.00 \\
\hline Nausea and vomiting & 0.00 & 0.00 & 16.67 & 100.00 \\
\hline Pain & 0.00 & 0.00 & 33.33 & 100.00 \\
\hline Dyspnea & 0.00 & 0.00 & 33.33 & 100.00 \\
\hline Insomnia & 0.00 & 0.00 & 33.33 & 100.00 \\
\hline Appetite loss & 0.00 & 0.00 & 33.33 & 100.00 \\
\hline Cough & 0.00 & 0.00 & 0.00 & 100.00 \\
\hline Diarrhea & 0.00 & 0.00 & 0.00 & 100.00 \\
\hline Financial problems & 0.00 & 33.33 & 66.67 & 100.00 \\
\hline
\end{tabular}

${ }^{1}$ Quartile is a numerical value in which all values are arranged from small to large in statistics and are divided into four equal positions. 2nd quartile is the median of all the values in the sample.

Table 4 The results of a multilevel ordinal logistic regression analysis of global health.

\begin{tabular}{lccc}
\hline Factor & OR & $P$ & $\mathbf{9 5 \%} \boldsymbol{C I}$ \\
\hline Education & & & \\
$\quad$ Junior /high school or above & 1.44 & 0.003 & $1.13 \sim 1.83$ \\
$\quad$ Primary school or below & 1.00 & & \\
Nutritional support & & & \\
$\quad$ Yes & 1.91 & 0.000 & $1.52 \sim 2.41$ \\
No & 1.00 & & \\
PG-SGA & & & \\
$0 \sim 1$ & 4.85 & 0.000 & $3.10 \sim 7.59$ \\
$2 \sim 3$ & 2.79 & 0.000 & $1.89 \sim 4.11$ \\
$4 \sim 8$ & 2.30 & 0.000 & $1.71 \sim 3.08$ \\
$\geq 9$ & 1.00 & & \\
\hline
\end{tabular}

Explanatory variables of the qoL: results of the multilevel ordinal logistic regression analysis

The results of the multilevel ordinal logistic regression analysis for global health are presented in Table 4 . The total model likelihood ratio test is a comparative model that uses only the intercept. In our analysis, the -2 Log Likelihood decreased from 4072.715 to $3900.669\left(\chi^{2}=172.046, P\right.$ $<0.0001)$, indicating that at least one overall regression 
coefficient of the independent variables was non- 0 , and the model was a good fit. The general quality of life among patients who had a junior /high school or above-level education was higher than that of the patients with primary school or lower-level education, with an $O R$ value of 1.44.

The measurements for the 14 dimensions were all statistically compared. The impact of each item on the functional domains is shown in Table 5. Only items that had a significant impact on the functional domains of QoL were included. The impact of cancer treatments on patient outcomes and their quality of life was already wellrecognized, and not surprisingly was confirmed in our population (functional domains $O R$ : 2.38, 2.19, 1.47, 1.48, 1.54). In addition, we found that age has an impact on the PF, EF and CF (OR: 1.40, 0.80 and 1.27); occupation has a significant effect on the PF, EF, and CF (OR: 1.93, 2.38, 2.68); and nutritional support (OR: 2.61, 3.55, 1.92, 1.96 and 1.88) and the PG-SGA score (OR: 4.75, 4.84, 2.12, 2.81 and 3.00) have significant effects on the PF, RF, EF, CF and $\mathrm{SF}$ functional domains. Indicators that had a significant impact on symptom domains are shown in Table 6 .

Table 5 The significance of factors based on a multilevel ordinal logistic regression analysis for the functional domains.

\begin{tabular}{|c|c|c|}
\hline Variable & OR $(95 \% C I)$ & $P$ \\
\hline \multicolumn{3}{|l|}{ Physical } \\
\hline \multicolumn{3}{|l|}{ Age } \\
\hline$<65$ years & $1.40 \quad(1.12 \sim 1.74)$ & 0.003 \\
\hline$\geq 65$ years & 1.00 & \\
\hline \multicolumn{3}{|l|}{ Gender } \\
\hline Female & $0.65 \quad(0.49 \sim 0.86)$ & 0.003 \\
\hline Male & 1.00 & \\
\hline \multicolumn{3}{|l|}{ Occupation } \\
\hline Worker & $1.93(1.19 \sim 3.15)$ & 0.014 \\
\hline Farmer & 1.00 & \\
\hline \multicolumn{3}{|l|}{ Cancer treatment } \\
\hline Other & $2.38 \quad(1.79 \sim 3.16)$ & 0.000 \\
\hline Chemo + surgery & 1.00 & \\
\hline \multicolumn{3}{|l|}{ Nutritional support } \\
\hline Yes & $2.61 \quad(2.07 \sim 3.29)$ & 0.000 \\
\hline No & 1.00 & \\
\hline \multicolumn{3}{|l|}{ PG-SGA } \\
\hline $0 \sim 1$ & $4.75 \quad(2.85 \sim 5.63)$ & 0.000 \\
\hline $2 \sim 3$ & $3.30 \quad(2.11 \sim 4.37)$ & 0.000 \\
\hline $4 \sim 8$ & $3.01 \quad(2.14 \sim 3.84)$ & 0.000 \\
\hline$\geq 9$ & 1.00 & \\
\hline \multicolumn{3}{|l|}{ Role } \\
\hline \multicolumn{3}{|l|}{ Cancer treatment } \\
\hline Other & $2.19(1.62 \sim 2.96)$ & 0.000 \\
\hline Chemo-radiotherapy & $1.41 \quad(1.05 \sim 1.88)$ & 0.020 \\
\hline Chemo + surgery & 1.00 & \\
\hline
\end{tabular}

Table 5 (Continued)

\begin{tabular}{|c|c|c|c|}
\hline \multicolumn{4}{|l|}{ Health insurance } \\
\hline Self-pay & 0.68 & $(0.48 \sim 0.95)$ & 0.025 \\
\hline Medical insurance & & 1.00 & \\
\hline \multicolumn{4}{|l|}{ Nutritional support } \\
\hline Yes & 3.55 & $(2.77 \sim 4.54)$ & 0.000 \\
\hline No & & 1.00 & \\
\hline \multicolumn{4}{|l|}{ PG-SGA } \\
\hline $0 \sim 1$ & 4.84 & $(2.70 \sim 8.67)$ & 0.000 \\
\hline $2 \sim 3$ & 2.36 & $(1.52 \sim 3.67)$ & 0.000 \\
\hline $4 \sim 8$ & 2.45 & $(1.76 \sim 3.42)$ & 0.000 \\
\hline$\geq 9$ & & 1.00 & \\
\hline
\end{tabular}

Emotional

Age

$<65$ years

$\geq 65$ years

$0.80 \quad(0.64 \sim 0.99)$

0.049

1.00

Occupation

Worker

Retiree + others

Farmer

$2.38(1.45 \sim 3.92) \quad 0.001$

$1.54(1.17 \sim 2.03) \quad 0.002$

Cancer treatment

Other

Chemo-radiotherapy

Chemo + surgery

1.00

$1.47(1.11 \sim 1.95) \quad 0.007$

$1.57(1.19 \sim 2.08) \quad 0.001$

1.00

Nutritional support

Yes

$1.92(1.52 \sim 2.43) \quad 0.000$

No

1.00

PG-SGA

$0 \sim 1$

$2.12(1.32 \sim 3.41) \quad 0.002$

$2 \sim 3$

$4 \sim 8$

$\geq 9$

Cognitive

Age

$<65$ years

$\geq 65$ years

$1.27(1.01 \sim 1.6) \quad 0.044$

1.00

Occupation

Worker

$2.68(1.54 \sim 4.66) \quad 0.000$

Farmer

1.00

Cancer treatment

Other

Chemo + surgery

1.48 (1.10 1.99)

0.010

1.00

Nutritional support

Yes

$1.96(1.54 \sim 2.50)$

0.000

1.00

PG-SGA

$0 \sim 1$

$2.81(1.62 \sim 4.87) \quad 0.000$

$2 \sim 3$

$2.06(1.32 \sim 3.20) \quad 0.001$ 
Table 5 (Continued)

\begin{tabular}{lcc}
\hline $4 \sim 8$ & $2.23(1.59 \sim 3.13)$ & 0.000 \\
$\geq 9$ & 1.00 & \\
Social & & \\
Cancer treatment & & \\
$\quad$ Other & $1.54(1.16 \sim 2.05)$ & 0.003 \\
$\quad$ chemotherapy + surgery & 1.00 & \\
Health insurance & & \\
Self-pay & $0.67(0.48 \sim 0.93)$ & 0.017 \\
Medical insurance & 1.00 & \\
Nutritional support & & \\
Yes & $1.88(1.48 \sim 2.38)$ & 0.000 \\
No & 1.00 & \\
PG-SGA & & \\
$0 \sim 1$ & $3.00(1.85 \sim 4.63)$ & 0.000 \\
$2 \sim 3$ & $2.12(1.73 \sim 2.92)$ & 0.000 \\
$4 \sim 8$ & $1.92(1.40 \sim 2.58)$ & 0.000 \\
$\geq 9$ & 1.00 & \\
\hline
\end{tabular}

Note: we analyzed the impact of all factors on quality of life, but only listed meaningful groups in each functional domain.

Table 6 The significance of factors after a multilevel ordinal logistic regression analysis for the symptom domains.

\begin{tabular}{|c|c|c|}
\hline Variable & $O R(95 \% C I)$ & $P$ \\
\hline \multicolumn{3}{|l|}{ Fatigue } \\
\hline \multicolumn{3}{|l|}{ Gender } \\
\hline Female & 1.39 (1.04 1.84) & 0.024 \\
\hline Male & 1.00 & \\
\hline \multicolumn{3}{|l|}{ Occupation } \\
\hline Worker & $0.61(0.38 \sim 0.99)$ & 0.046 \\
\hline Farmer & 1.00 & \\
\hline \multicolumn{3}{|l|}{ Cancer treatment } \\
\hline Other & $0.46(0.34 \sim 0.60)$ & 0.000 \\
\hline Chemo + surgery & 1.00 & \\
\hline \multicolumn{3}{|l|}{ Nutritional support } \\
\hline Yes & $0.47(0.37 \sim 0.59)$ & 0.000 \\
\hline No & 1.00 & \\
\hline \multicolumn{3}{|l|}{ PG-SGA } \\
\hline $0 \sim 1$ & $0.24(0.15 \sim 0.38)$ & 0.000 \\
\hline $2 \sim 3$ & $0.27(0.18 \sim 0.41)$ & 0.000 \\
\hline $4 \sim 8$ & $0.37(0.27 \sim 0.50)$ & 0.000 \\
\hline$\geq 9$ & 1.00 & \\
\hline \multicolumn{3}{|l|}{ Nausea and vomiting } \\
\hline \multicolumn{3}{|l|}{ Gender } \\
\hline Female & $1.46(1.03 \sim 2.08)$ & 0.033 \\
\hline Male & 1.00 & \\
\hline \multicolumn{3}{|l|}{ Occupation } \\
\hline Retiree + others & $0.61(0.43 \sim 0.88)$ & 0.007 \\
\hline
\end{tabular}

Table 6 (Continued)

\begin{tabular}{lcc}
\hline Farmer & 1.00 & \\
Cancer treatment & & \\
Other & $0.49(0.34 \sim 0.73)$ & 0.000 \\
Chemo + surgery & 1.00 & \\
Nutritional support & & \\
Yes & $0.53(0.40 \sim 0.70)$ & 0.000 \\
No & 1.00 & \\
PG-SGA & & \\
$0 \sim 1$ & $0.31(0.14 \sim 0.64)$ & 0.002 \\
$2 \sim 3$ & $0.32(0.17 \sim 0.62)$ & 0.001 \\
$4 \sim 8$ & $0.29(0.18 \sim 0.47)$ & 0.000 \\
$\geq 9$ & 1.00 & \\
Pain & & \\
Cancer treatment & & \\
Other & $0.58(0.43 \sim 0.79)$ & 0.001 \\
Chemo + surgery & 1.00 & \\
Nutritional support & & 0.000 \\
Yes & $0.57(0.45 \sim 0.72)$ & \\
No & 1.00 &
\end{tabular}

PG-SGA

$\begin{array}{ccc}0 \sim 1 & 0.24(0.13 \sim 0.44) & 0.000 \\ 2 \sim 3 & 0.35(0.22 \sim 0.51) & 0.000 \\ 4 \sim 8 & 0.45(0.32 \sim 0.64) & 0.000 \\ \geq 9 & 1.00 & \end{array}$

Dyspnea

Cancer treatment

$\begin{array}{lcc}\text { Other } & 0.39(0.25 \sim 0.56) & 0.000 \\ \text { Chemo-radiotherapy } & 0.62(0.41 \sim 0.86) & 0.005 \\ \text { Chemo + surgery } & 1.00 & \end{array}$

Nutritional support

Yes
No
PG-SGA
$0 \sim 1$
$\geq 9$

$0.54(0.41 \sim 0.72) \quad 0.000$

1.00

$0.46(0.22 \sim 0.93) \quad 0.030$

1.00

Insomnia

Gender

$\begin{array}{lcc}\text { Female } & 1.37(1.01 \sim 1.86) & 0.043 \\ \text { Male } & 1.00 & \end{array}$

Permanent residence
Urban
Rural

1.49 (1.15 1.94)

0.003

1.00

Occupation

Worker

$0.56(0.33 \sim 0.97)$

0.037

Farmer

1.00

Nutritional support 


\begin{tabular}{|c|c|c|c|c|c|}
\hline No & 1.00 & & Rural & 1.00 & \\
\hline PG-SGA & & & Education & & \\
\hline $0 \sim 1$ & $0.29(0.16 \sim 0.52)$ & 0.000 & Primary school or below & $0.57(0.35 \sim 0.92)$ & 0.023 \\
\hline $2 \sim 3$ & $0.51(0.32 \sim 0.81)$ & 0.004 & University & 1.00 & \\
\hline $4 \sim 8$ & $0.55(0.39 \sim 0.77)$ & 0.001 & Occupation & & \\
\hline$\geq 9$ & 1.00 & & Cadre & $0.65(0.45 \sim 0.94)$ & 0.021 \\
\hline Appetite loss & & & Worker & $0.52(0.32 \sim 0.85)$ & 0.008 \\
\hline Occupation & & & Retiree + others & $0.56(0.42 \sim 0.74)$ & 0.000 \\
\hline Worker & $0.56(0.31 \sim 0.99)$ & 0.048 & Farmer & 1.00 & \\
\hline Farmer & 1.00 & & Cancer treatment & & \\
\hline Cancer treatment & & & Other & $0.69(0.52 \sim 0.92)$ & 0.011 \\
\hline Other & $0.41(0.29 \sim 0.58)$ & 0.000 & Surgery & $0.68(0.48 \sim 0.96)$ & 0.026 \\
\hline Chemo + surgery & 1.00 & & Chemo + surgery & 1.00 & \\
\hline Nutritional support & & & PG-SGA & & \\
\hline Yes & $0.55(0.42 \sim 0.71)$ & 0.000 & $0 \sim 1$ & $0.37(0.23 \sim 0.58)$ & 0.000 \\
\hline No & 1.00 & & $2 \sim 3$ & $0.38(0.25 \sim 0.57)$ & 0.000 \\
\hline PG-SGA & & & $4 \sim 8$ & $0.49(0.36 \sim 0.67)$ & 0.000 \\
\hline $0 \sim 1$ & $0.16(0.08 \sim 0.33)$ & 0.000 & $\geq 9$ & 1.00 & \\
\hline
\end{tabular}

$2 \sim 3$

$4 \sim 8$

$\geq 9$

Cough

Gender

Male

Female

$1.47(1.00 \sim 2.15) \quad 0.048$ 1.00

Nutritional support

Yes
No

$0.59(0.43 \sim 0.81)$

0.001

1.00

PG-SGA

$0 \sim 1$

2 3

$4 \sim 8$

$\geq 9$

Diarrhea

Cancer treatment

Other
Chemo-radiotherapy
Chemo + surgery
PG-SGA
$0 \sim 1$
$\geq 9$

$0.16(0.05 \sim 0.52) \quad 0.002$

$0.42(0.23 \sim 0.56) \quad 0.014$

$0.31(0.18 \sim 0.55) \quad 0.000$

1.00

Financial problems

Age

$<65$ years

$1.56(1.25 \sim 1.96)$

0.000

$\geq 65$ years

1.00

Permanent residence

Urban
$0.38(0.22 \sim 0.66) \quad 0.001$

$0.42(0.25 \sim 0.71) \quad 0.001$

1.00

0.027

1.00
$0.20(0.05 \sim 0.83)$

Note: we analyzed the impact of all factors on symptom domains of QoL, but only listed meaningful groups to avoid the complexity of the table.

\section{Discussion}

Quality of Life is a multidimensional concept emphasizing the subjective feelings and functional status of patients, with dimensions encompassing physical, social, and psychological functioning [12]. Previous studies have shown that the physical, societal, emotional and other functions of esophageal cancer patients were significantly decreased, and their quality of life was lower than that of healthy subjects [13]. Although it is well-known that symptoms related to esophageal cancer, such as dysphagia, reflux, eating difficulties, diarrhea, appetite loss, are contributors to a poorer QoL [14], the relative influence of other factors, such as the patient demographics and socioeconomic status, have rarely been evaluated. To assess the importance of these factor and implement further targeted interventions, we evaluated the relationships between the nutritional status, demographic information, and socioeconomic variables to all dimensions of the QoL in individuals with esophageal cancer.

Previous studies suggested that $50 \% \sim 90 \%$ of oncology patients have some degree of malnutrition [15]. In the current study, the PG-SGA revealed that only $6.0 \%$ of the patients were well-nourished, which was consistent with the results reported by Quyen TC et al. [16], indicating that esophageal cancer patients are more likely to suffer from malnutrition than those with other types of cancers. Decreased dietary intake, metabolic changes, cancer cachexia, and esophageal cancer-related symptoms may synergistically contribute to cancer-related malnutrition in cancer patients [17]. Lis CG et al. demonstrated a strong associa- 
tion between the nutritional status and QoL in patients with head and neck cancer, gastrointestinal cancer, lung cancer and gynecological cancer by reviewing extensive available literature [18]. The results of our current ordinal logistic regression analysis showed that malnutrition is one of the main causes of the decline in QoL among esophageal cancer patients. Not surprisingly, the QoL of the well-nourished patients was higher than that of the patients who were malnourished (as defined by PG-SGA score $>2$ ).

We then analyzed the potential influence of nutritional support. The results demonstrated that patients who were provided with nutritional support had better PF, RF, CF, EF and SF scales, with OR values ranging from 1.86 to 3.50 , and these patients were less likely to have high symptomatic risk (except for diarrhea and economic difficulties) than those without nutritional support (OR: 0.45 - 0.58). Senesse $\mathrm{P}$ et al. [19] also found that home parenteral nutrition (HPN) could benefit the quality of life for malnourished patients with gastrointestinal cancer. In patients with advanced cancer, the PG-SGA is a reliable and effective nutritional screening index, and has the advantages of being a rapid, noninvasive and easy-to-perform test to dynamically evaluate the nutritional status of patients.

A few studies have suggested that a poor QoL is associated with poorer long-term survival in esophageal cancer patients [20]. Additionally, clinicians must take the patients' perception of their QoL into consideration. Decision-making and the management of esophageal cancer would be best performed by a multidisciplinary committee and should be personalized for the specific circumstances of each case $[21,22]$. Identifying the factors that influence the patient QoL and then giving the necessary treatment and nursing measures are vitally important for patients with esophageal cancer [23].

There are a few limitations of this study that require attention or improvements. The cross-sectional nature of this project makes it impossible to make any causal inferences regarding the relationship between malnutrition and QoL. Further studies are needed to assess whether the QoL and the survival rate of the patients with esophageal cancer were improved after nutritional intervention. Secondly, there were significantly more men $(n=1009)$ than women $(n$ $=214$ ) in this study. In 2016, the National Central Cancer Registry (NCCR) collected registration data for 2013 from local cancer registries to estimate the cancer incidence rates in China. The age-standardized incidence rate of esophageal cancer in China was 13.82/100,000 (20.14/100,000 men and $7.65 / 100,000$ women). Therefore, there is a certain selection bias in this study due to the gender-specific incidence rates.

In summary, we found that nutritional support and the PG-SGA score variables were significantly associated with the QoL in patients with esophageal cancer.

\section{Acknowledgements}

We thank all of the members of the study institutions for their help.

\section{Conflict of Interests}

The authors declare no conflict of interest.

\section{Funding}

This research was supported by research grants from the General Program of National Natural Science Foundation in China (No. 81373046) and the national Key Research and Development Program (No. 2017YFC1309200).

\section{References}

1. Thrift AP. The epidemic of esophageal carcinoma: Where are we now? Cancer Epidemiol 2016;41:88-95.

2. Nordenstedt H, El-serag H. The influence of age, sex, and race on the incidence of esophageal cancer in the United States (1992-2006). Scand J Gastroentero 2011;46(5):597-602.

3. Wheeler JB, Reed CE. Epidemiology of esophageal cancer. Surg Clin North Am 2012;92(5):1077-87.

4. Chen W, Sun K, Zheng R, Zeng H, Zhang S, Xia C, Yang Z, Li H, Zou X, He J. Cancer incidence and mortality in China, 2014. Chin J Cancer Res 2018;30(1):1-12.

5. Encinas de la Iglesia J, Corral de la Calle MA, Fernández Pérez GC, Ruano Pérez R, Álvarez Delgado A. Esophageal cancer: anatomic particularities, staging, and imaging techniques. Radiologia 2016;58(5):352-65.

6. Alsop BR, Sharma P. Esophageal cancer. Gastroenterol Clin North Am 2016;45(3):399-412.

7. Darling GE. Quality of life in patients with esophageal cancer. Thorac Surg Clin 2013; 23(4):569-75.

8. Fayers P, Bottomley A. EORTC Quality of Life Group; Quality of Life Unit. Quality of life research within the EORTC - the EORTC QLQ-C30. Eur J Cancer 2002;Suppl 4:S125-33.

9. Blazeby JM, Farndon JR, Donovan J, Alderson D. A prospective longitudinal study examining the quality of life of patients with esophageal carcinoma. Cancer 2000;88(8):1781-7.

10. Djärv T, Metcalfe C, Avery KN, Lagergren P, Blazeby JM. Prognostic value of changes in health-related quality of life scores during curative treatment for esophagogastric cancer. J Clin Oncol 2010;28(10):1666-70.

11. Ottery FD. Rethinking nutritional support of the cancer patient: the new field of nutritional oncology. Semin Oncol 1994;21(6):770-8.

12. Testa MA, Simonson DC. Assessment of quality-of-life outcomes. N Engl J Med 1996;334(13):835-40.

13. Zhu LL, Li XM. [Research progress on the quality of life in patients with esophageal cancer]. J Nurs Admin 2011;12:857-9.

14. Schandl A, Lagergren J, Johar A, Lagergren P. Health-related quality of life 10 years after oesophageal cancer surgery. Eur J Cancer 2016; 69:43-50.

15. Bozzetti F. Nutritional support of the oncology patient. Crtic Rev 
Oncol Hematol 2013;87(2):172-200.

16. Quyen TC, Angkatavanich J, Thuan TV, Xuan VV, Tuyen LD, Tu DA. Nutrition assessment and its relationship with performance and Glasgow prognostic scores in Vietnamese patients with esophageal cancer. Asia Pac J Clin Nutr 2017; 26(1):49-58.

17. Tong $\mathrm{H}$, Isenring E, Yates $\mathrm{P}$. The prevalence of nutrition impact symptoms and their relationship to quality of life and clinical outcomes in medical oncology patients. Support Care Cancer 2009;17(1):83-90.

18. Lis CG, Gupta D, Lammersfeld CA, Markman M, Vashi PG. Role of nutritional status in predicting quality of life outcomes in cancer - a systematic review of the epidemiological literature. Nutr J 2012;11:27.

19. Senesse P, Tadmouri A, Culine S, Dufour PR, Seys P, Radji A, Rotarski M, Balian A, Chambrier C. A prospective observational study assessing home parenteral nutrition in patients with gastroin- testinal cancer: benefits for quality of life. J Pain Symptom Manage 2015;49(2):183-91.

20. Blazeby JM, Brookes ST, Alderson D. Prognostic value of quality of life scores in patients with oesophageal cancer. Br J Surg 2000;87(3):362-73.

21. Lordick F, Mariette C, Haustermans K, Obermannová R, Arnold D; ESMO Guidelines Committee. Oesophageal cancer: ESMO Clinical Practice Guidelines for diagnosis, treatment and follow-up. Ann Oncol 2016;27(suppl 5): v50-7.

22. D'Journo XB, Thomas PA. Current management of esophageal cancer. J Thorac Dis 2014;6 (Supp1 2):S253-64.

23. Du LB, Li HZ, Wang YQ, Zhu C, Zheng RS, Zhang SW, Chen WQ, He J. [Report of colorectal cancer incidence and mortality in China, 2013]. Zhonghua Zhong Liu Za Zhi 2017; 39(9):701-6. 